\title{
1023
}

\section{Estimation of Water Content in Partially Saturated Soil Horizons with Ground-penetrating Radar}

\author{
E. Strobach* (Curtin University), B.D. Harris (Curtin University), A.W. Kepic \\ (Curtin University), J.D. Dupuis (Curtin University) \& M.W. Martin (Water \\ Corp., Perth)
}

\section{SUMMARY}

Ground-penetrating radar (GPR) measurements have been used to map the absolute water content of water retentive layers found within the vadose zone of the superficial aquifer at the Gnangara Mound near Perth, Western Australia. The lateral water content distribution was derived from a pseudo 3D GPR dataset acquired over a podzolic soil profile. The water content of the bulk vadose zone was estimated from travel times. We discuss the parameters that influence the quantification of water content within a water retentive layer. By constraining key variables as layer thickness, porosity, Lichteneker-Rother model parameters, background permittivity and point of reflection, we can make a reasonable quantification of water content in the water retentive soil horizon. Calculated water content is shown to lie within reported values. The horizons were mapped laterally and reveal large variations throughout the test site. Our GPR derived hydrogeologic model is confirmed by time-lapse neutron logs and a high-resolution electrical resistivity image. Finally we compare results with total reflected energy from the interval above the regional water table and obtain a good correlation with the water content map. 


\section{Introduction}

The Gnangara Mound is situated north of Perth, Western Australia. Its aquifers currently constitute Perth's most important water resource (Davidson, 1995). Water extraction and successive drawdown from the superficial aquifer have the potential to stress the ecosystems and the native bushland. To this effect, and to meet the water needs of the expanding population of Perth, the Water Corporation and the Department of Water of Western Australia have undertaken a study of the interaction between water dependent ecosystems, soils and water table fluctuations. The recharge potential of the Gnangara Mound is in part governed by vertical water flux mechanisms for different soils (Xu et al., 2008). In order to investigate the distribution and the potential impact of water retentive layers on local ecosystems and groundwater recharge, hundreds of line kilometres of ground-penetrating radar have been acquired over important regions of recharge (Strobach et al., 2010).
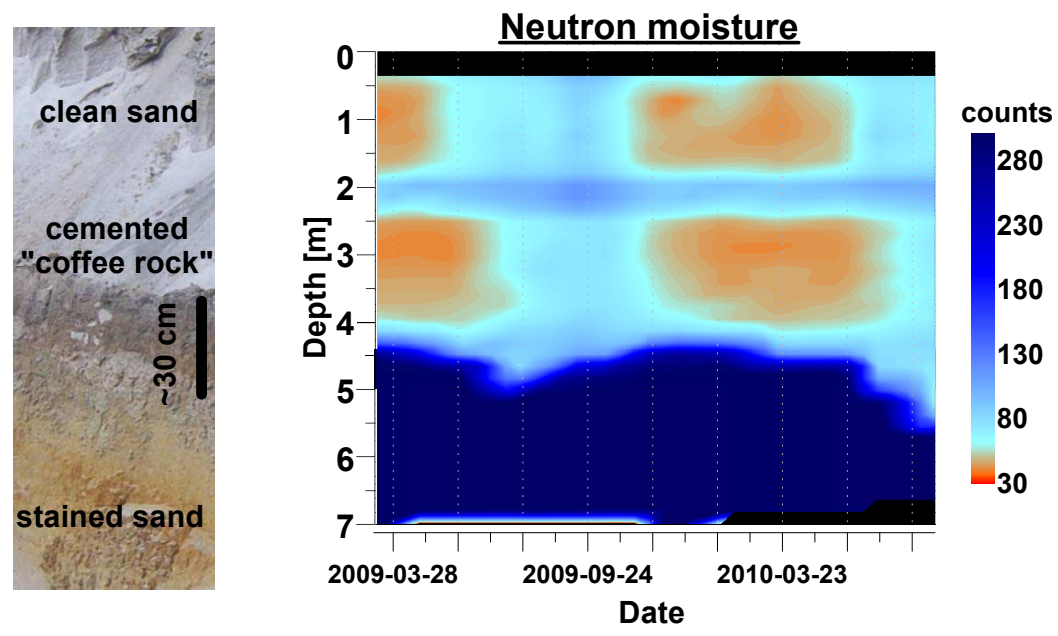

Figure 1 Left: field example of "Coffee Rock"; Right: gridded time-lapse Neutron counts, measurements were collected monthly and data was gridded (kriging) to 15-day intervals. Data provided by Water Corporation

Previous studies by Froend (pers. comm.) suggest that some water retentive soil horizons found within the podzolic soil profiles (Podosols) sequester water during the wet season which can then be available to vegetation throughout the dry season. These layers have been referred to as "coffee rock" due to their brown coloration (Fig. 1, left). These layers vary in degree of cementation and are spatially discontinuous on a kilometre scale and sometimes on a 10 meter scale (Strobach et al., 2010). They have been described as loamy, light to dark brown, from strongly cemented to friable sand (Bertuch and Froend, 2006). Yellow stained sand, covered by iron hydroxide precipitate, is typically found below the cemented "coffee rock" horizon (Fig. 1).

GPR and ERI measurements were conducted at Whiteman Park, close to a vegetation monitoring site (Bertuch and Froend, 2006). Time-lapse gravimetric water content measurements (Froend, pers. comm.) and monthly borehole neutron logs reveal a water retentive horizon (Fig. 1, right). Bertuch and Froend (2006) describe a loamy dark brown sand horizon at $2 m$ depth. Its water-retentiveness is inferred from the time-lapse gravimetric water content measurements and Neutron logs (Fig. 1, right) where higher counts are observed within the interval between $1.7 \mathrm{~m}$ and $2.3 \mathrm{~m}$ throughout the summer period (November to March). This is in contrast with the clean sand interval where the gravimetric water contents range between $0 \%$ (after summer) to 5\% (winter) for the clean sand strata whilst the the water retentive horizon stores between 5\% (after summer) and 12\% (winter) (Froend, pers. comm.).

\section{Methods}

The pseudo 3D GPR data were acquired as a series of 2D common-offset gathers triggered in time. Shielded antennas with a centre frequency of $250 \mathrm{MHz}$ were used in conjuction with a real time kine- 
matic differential GPS which provided the needed spatial information. The dataset acquired over an area of $25 \times 50 \mathrm{~m}$ consists of 25 lines with an inter-line spacing of approximately $1 \mathrm{~m}$. Static corrections for the air-wave arrival and a DC-shift were applied to the data before picking of the layer. This limited processing on the data was done in order to preserve relative amplitudes and the time signatures of the reflections. Further processing followed prior to gridding of the time-slices (Fig. 2a) that were migrated, topography corrected and had a 2D median filter applied (cp. Fig. 3 upper). The water content within

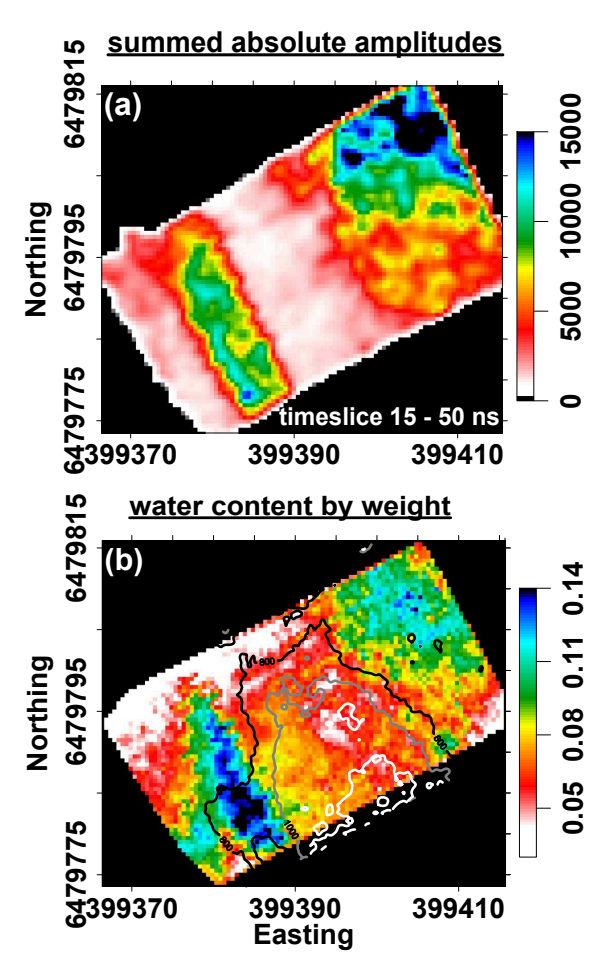

Figure 2 (a) cumulative absolute amplitudes of interval between 15 and $50 \mathrm{~ns}$ (distribution of layers in vadose zone); (b) water content map for a $0.4 \mathrm{~m}$ thick layer, contour lines are the gridded maximum envelope values of the water table reflection

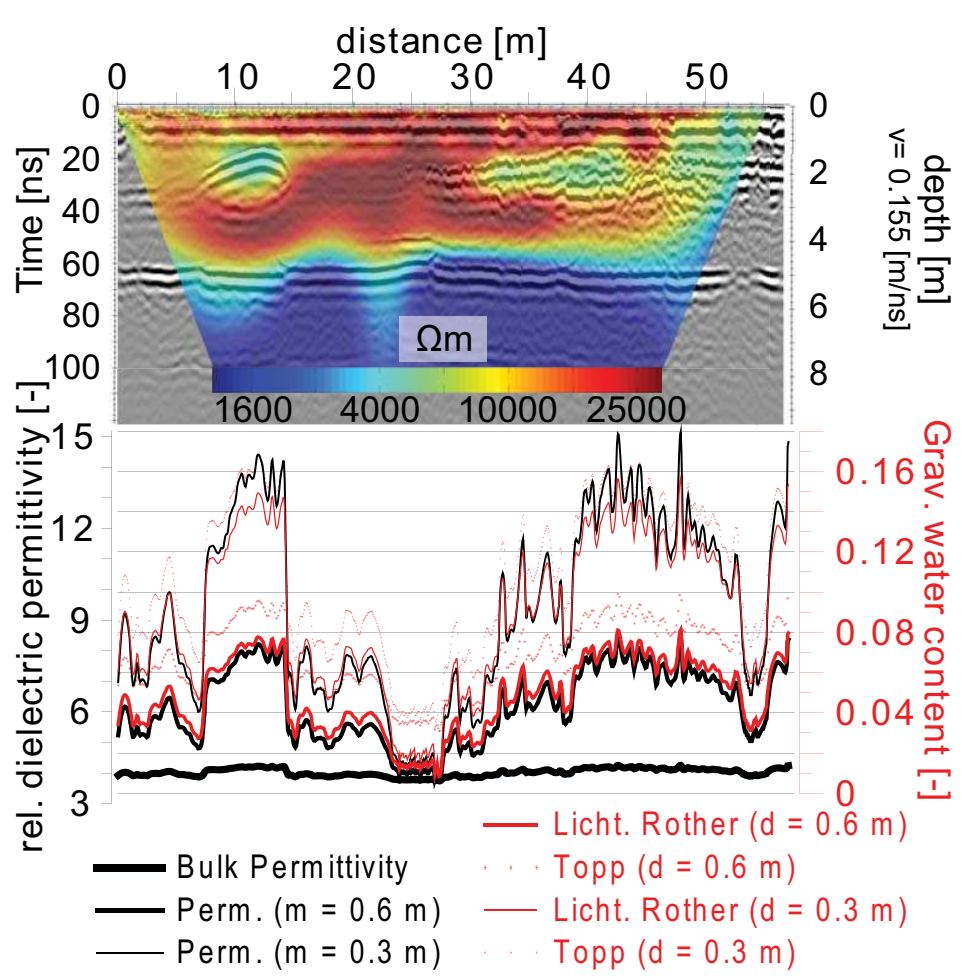

Figure 3 Upper: migrated GPR transect with ERI overlay (data inverted with DC2dInvRes by Thomas Günther, www.resistivity.net), dry sands, retentive layers and water table show resistivities of $10-30 \mathrm{k} \Omega \mathrm{m}, 2-5 \mathrm{k} \Omega \mathrm{m}$ and $\sim 100 \Omega \mathrm{m}$, respectively; Lower: permittivity (black) and calculated grav. water content (red) profiles for various layer thicknesses, comparison of Lichteneker-Rother (solid) and Topp-model (dotted)

porous sediments influence the velocity of propagation of the propagating electromagnetic wave used for imaging with GPR (Huisman et al., 2003; Gloaguen et al., 2001). Thus, a change in velocity of propagation allows us to infer a change in the water content found amongst the sediments. This is observed as a reflection time anomaly, where the water table reflection is pushed towards a later time (e.g. below Anomaly A in figure 3 upper). From these travel time anomalies we can attempt to quantify the velocity contrast and hence water content of a layer needed to induce the measured time shift assuming that the solid phase is otherwise homogeneous. In the first order, this method provides an average water content for the vadose zone above the water table. The water content estimates are calibrated by first identifying an area along the transect where the sediments above the water table are homogeneous. The depth to the water table is calculated by using the travel time to the water table and the measured background velocity from diffraction hyperbolas present in the datasets $\left(v_{0} \sim 0.155 \frac{\mathrm{m}}{\mathrm{ns}}\right)$. Taking into account the topographic variations, we assumed the water table reflection to be at a constant position throughout the test-site and and thus can calculate a depth $D$ at each point. The inferred position compares well with borehole data nearby (e.g. saturated zone in figure 1 right). By picking two-way travel times along the water table reflection, layer velocities were assumed to sum up in series, therefore total two-way-traveltime 


$$
t_{t w t}=\sum_{i} t_{i}=2 \sum_{i} \frac{d_{i}}{v_{i}}=\frac{2}{c} \sum_{i} d_{i} \varepsilon_{r, i}
$$

where $d_{i}, v_{i}$ are thickness and velocity of the layer $i$, respectively, and $c$ the speed-of-light in vacuum. For a two-layer case (background medium and retentive layer) we obtain for the travel time through a layer

$$
t_{d}=2 \frac{D-d}{v_{0}}+\frac{d}{v_{d}}=\frac{2}{c}\left((D-d) \sqrt{\varepsilon_{r, 0}}+d \sqrt{\varepsilon_{r, d}}\right) \quad \text { and } \quad v_{d}=\frac{2 d}{t_{d}-\frac{2(D-d)}{v_{0}}} \quad \text { or } \quad \varepsilon_{r, d}=\frac{c^{2}}{v_{d}^{2}}
$$

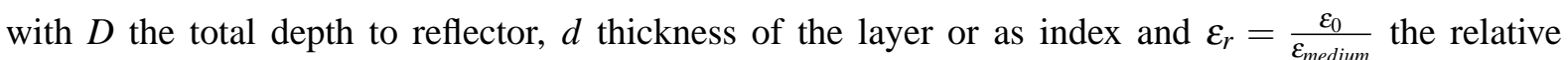
dielectric permittivity. To link layer permittivity and volumetric water content, we used the LichtenekerRother model for a three-phase mix of solid matrix, water and air. Assuming a porosity of $40 \%$, the Lichteneker-Rother relationship (Brovelli and Cassiani, 2008) thus has effectively two fitting parameters which are the solid matrix permittivity $\varepsilon_{r, s}$ and the exponent $\alpha$, and volumetric water content becomes

$$
\theta_{v}=\frac{\phi\left(\varepsilon_{r, a i r}^{\alpha}-\varepsilon_{r, s}^{\alpha}\right)-\varepsilon_{r, d}^{\alpha}+\varepsilon_{r, s}^{\alpha}}{\varepsilon_{r, a i r}^{\alpha}-\varepsilon_{r, H_{2} O}^{\alpha}}
$$

with $\varepsilon_{r, a i r}, \varepsilon_{r, s}, \varepsilon_{r, H_{2} O}$ and $\varepsilon_{r, d}$ the relative dielectric permittivities of air (1), the solid phase (here assumed to be 5.5), water (80) and of the bulk layer with thickness $d$, respectively. $\alpha$ was set to 0.55 . The choices of $\alpha$ and $\varepsilon_{r, s}$ are justified as we can expect a slightly higher permittivity for the solids and the $\alpha$-value as the layer is cemented and iron hydroxides and clay minerals are present. Those minerals complicate the relationship between bulk electromagnetic properties and water content for unsaturated conditions. As pointed out by Brovelli and Cassiani (2008), the $\alpha$ exponent in the Lichteneker-Rother equation does not have a direct physical meaning, and they propose a new model which includes a cementation factor and a saturation exponent (Brovelli and Cassiani, 2010), which would allow for another degree of freedom. By varying $\alpha$, however, we acknowledge that the value of 0.5 (CRIM-model) is not a physical constant.

\section{Discussion of the results}

The main goal of the 3D dataset was to map lateral changes in layer properties (e.g. Fig. 3, marked as anomaly A and B, Fig. 2a). The test-site consists of three major areas. The central area shows no reflector and the soil profile most likely consists of clean sand without any major soil horizons. The mid-western part shows an elongated, north-south striking anomaly B at $1-1.5 \mathrm{~m}$ depth within the clean sand. It is characterized as a strong undisturbed reflection with diffractions originating at its sides. The travel-times of the water table reflection below anomaly A become greater while the reflection amplitude is not greatly influenced. In the eastern part we observe anomaly B as a strong reflector accompanied by many diffractions that disturb the radar image. Here, picking of the water table reflection was generally more difficult and travel times varied. Both, travel times and reflection amplitudes of the water table reflection are influenced in this area. We can distinguish three hypothetically contrasting recharge environments from very high recharge potential in areas without impeding layers, to lower recharge potential below anomalies A and B. Figure $2 \mathrm{~b}$ shows a map of gravimetric water contents calculated from travel times as described in the previous section. The contour lines plotted on top have been calculated by gridding the maximum of the envelope of the water table reflection. Here, we assumed a layer thickness of $0.4 \mathrm{~m}$ which will overestimate the water content where the layer thickness exceeds $0.4 \mathrm{~m}$. This thickness, however, does not only provide reasonable values for the water content, but has been chosen according to geologic and Neutron log observations. The three areas can be easily distinguished and identified. Anomaly B shows the greatest change in travel time, and thus water content, for the layer while area B has varying influence on the travel times. Note that water contents were calculated for a $0.4 \mathrm{~m}$ thick layer throughout the whole of the area and will greatly overestimate water contents where no layer is present. In figure 3 (lower) a permittivity (black lines) and water content (red lines) profile is plotted for a section running through the middle of the area. The gravimetric water contents 
were estimated from the volumetric water contents with the porosity of $40 \%$ and a particle density of $2.65 \frac{\mathrm{g}}{\mathrm{cm}^{3}}$ (quartz). We transformed from volumetric to gravimetric in order to compare to values reported by Froend. Note the slight difference between water contents obtained with the Topp-model (dotted lines) and the Lichteneker-Rother values (solid lines). As layer thickness $d$ is not precisely known and possibly varies throughout the test site, the velocity calculation is highly sensitive to this parameter, the Topp model would have been sufficient for a first-order estimate. Still, we used the Lichteneker Rother model to point out, that water content estimation from limited field observations without apriori knowledge is not unique. The map provided in figure 2 (b) should be understood as a representation of the total amount of water captured within a layer of variable thickness above the saturated zone. In order to quantify the GPR response of the retentive layers directly, we created a timeslice by summing absolute amplitudes within the interval of the retentive layers $(15-50 \mathrm{~ns})$ of the migrated 3D gridded dataset. Note the good correlation with the water contents from travel time analysis. The envelope contour lines in figure 2 (b), however, are not a powerful representation of the layers above.

\section{Conclusion}

The presented study demonstrates how GPR can provide valuable information on water retentive soil horizons. We successfully quantified lateral water content variations observed on a pseudo 3D GPR dataset and estimated associated moisture content within a layer of constant thickness within a homogeneous background. Values compare favorably with gravimetric and neutron log water content measurements. A high-resolution electrical resistivity image justifies the choice of our geologic model and complements the interpretation of the GPR. Too many parameters, however, are unknown to quantify the vertical water content distribution precisely. Namely porosity, layer thickness, relationship between bulk permittivity and water content (i.e. $\alpha$ exponent, solid phase or matrix permittivity $\varepsilon_{r, s}$ ) and the point of reflection had to be estimated, each with a considerable error. But, more importantly, we were able to demonstrate how variable those soil horizons are on a small scale, which will have a great impact on extrapolating water recharge environments to a larger scale.

\section{Acknowledgement}

We would like to thank Dominic Howman for assistance in the field. Elmar Strobach is funded by a Curtin University international postgraduate research scholarship and a student research grant by the Australian Society of Exploration Geophysics. The research was taken out under a Centre of High Definition Geophysics (CHDG) project and research grants provided by the Water Corporation.

\section{References}

Bertuch, M. and Froend, R. [2006] Winter drawdown trial - whiteman park, soil characterisation report. Tech. rep., Edith-Cowan University, School of Natural Sciences, Perth, Western Australia.

Brovelli, A. and Cassiani, G. [2010] A combination of the hashin-shtrikman bounds aimed at modelling electrical conductivity and permittivity of variably saturated porous media. Geophysical Journal International, 180(1), $225-237$.

Brovelli, A. and Cassiani, G. [2008] Effective permittivity of porous media; a critical analysis of the complex refractive index model. Geophysical Prospecting, 56(5), 715-727.

Davidson, W. [1995] Hydrogeology and groundwater resources of the Perth Region, Western Australia. Bulletin 142, Western Australia geological Survey.

Gloaguen, E., Chouteau, M., Marcotte, D. and Chapuis, R. [2001] Estimation of hydraulic conductivity of an unconfined aquifer using cokriging of gpr and hydrostratigraphic data. Journal of Applied Geophysics, 47(2), $135-152$.

Huisman, J.A., Hubbard, S.S., Redman, J.D. and Annan, A.P. [2003] Measuring soil water content with ground penetrating radar: A review. Vadose Zone J., 2(4), 476-491, doi:10.2113/2.4.476.

Strobach, E., Harris, B., Dupuis, J., Kepic, A. and Martin, M. [2010] Gpr for large-scale estimation of groundwater recharge distribution. Ground Penetrating Radar $(G P R), 2010$ 13th International Conference on, 1 -6, doi: 10.1109/ICGPR.2010.5550189.

Xu, C., Canci, M., Martin, M., Donnelly, M. and Stokes, R. [2008] Perth regional aquifer modelling system (prams) model development: Application of the vertical flux model. Department of Water, Western Australia, Hydrogeological record series, HG 27. 